\title{
Application of Neural Network Technology in Computer Communication
}

\author{
$\mathrm{Hu} \mathrm{Hai}^{1 \mathrm{a}}$, Li Xiao-ling ${ }^{2 b}$ \\ ${ }^{1}$ NanChang University, GongQing College \\ ${ }^{2}$ NanChang University, GongQing College \\ ${ }^{a}$ email, ${ }^{a}$ email
}

Key words: Neural network, Computer communication, Genetic algorithm

\begin{abstract}
The article combines with license plate recognition system, which states the application of computer communication based on the analysis of license plate recognition system based on neural network positioning system for analysis, based on related classification method and the work flow of neural network technique and so on. In addition, something about the genetic algorithm has also been studied. Then genetic algorithm and neural network technique were combined together. There is an analysis of computer communication application after a comprehensive analysis.
\end{abstract}

\section{Introduction:}

At present, most of pictures applied to license plate recognition system based on computer technique are taken by outdoor webcams. So it suffers a lot from external factors such as heavy rainfall, foggy weather, weak light intensity, man-made sabotage and spot on the license plate. All of which makes it difficult to position license plate accurately as numbers and letters are blurred. Therefore, this item which studies neural network positioning license plate system based on genetic algorithm is incrediblely necessary, and it not only has a lot more real-world meaning but also has a number of potential applications.

\section{Vehicle license Plate Identification of Computer Technology}

License plate recognition technique, as a key part of intelligent transportation system in China, has been widely applied to the fields of digital image technology, biometric identification technology and artificial intelligence technology. There are now wide and varied means of license plate recognition. What are widely used in practice include test of the characteristics, template match method and neural networks approaches. Or the combination of these methods will be applied to the systems of development and design. License plate recognition has gained widespread use. For example, the system is used by crime investigation division of police organ for detection of illegal vehicle parking, for test of vehicle driving violations, for stolen vehicle tracking. The system can be used by traffic administrative departments to estimate real-time traffic speed and to be applied in freeway toll station. What's more, both automatic charging system used by property management office and safe system about locomotive in and out are based on the license plate recognition technique.

At the moment, for most of the Vehicle license plate identification technology based on neural network, the first thing is to collect enough samples of license plate images as suspect images. Then the suspect images will be recognized with the help of BP neural network. There will be an end when it comes to the predetermined accuracy. Next license plate images that need recognition and location is to be pre-processed. Using image binaryzation and histogram processing reduce outside light that has 
affected the license plate images. In the next moment, background noise will be eliminated through the filter. The last thing is to transmit license plate images that have been pre-processed to the neural network research system in order to position license plates. The process toVehicle license plate identification technology based on neural network is illustrated in figure 1.

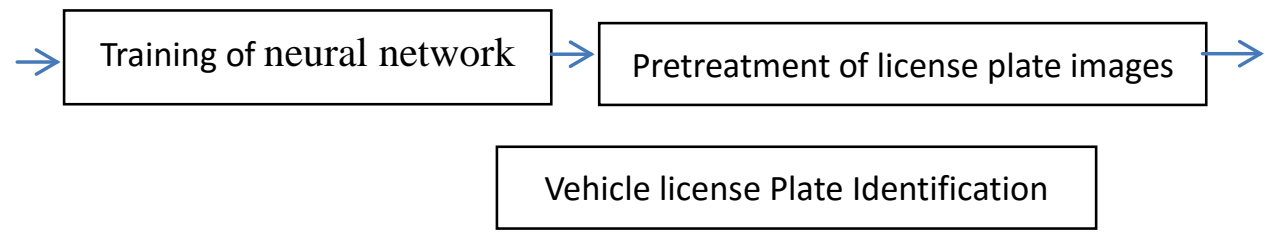

figure 1 The flow of Vehicle license Plate Identification based on neural network

\section{Introduction to Neural Network Technique}

There are two relatively common classifications of neural network. One classification is to follow the network connection topology. And another one is to follow the internal data flow of the network. Neural network based on the first classification consists of hierarchical structure and interconnection structure. Neurons in each layer from the network connect with each other but there is no connection in the same layer in Hierarchical structure, while each neuron from the network connects with each other in interconnection structure. And interconnection structure can be divided into sparse interconnection, partial interconnection and whole interconnection according to connection extent.

Neural network based on the internal data flow of the network can be divided into feed-forward neural network and feedback neural network. The structure of feed-forward neural network is the same as hierarchical structure. This means that all data flow needs to transfer from hidden layer to input layer layer by layer. And then data flow will be transferred to output layer but there is no feedback loop during the transmitting procedure. structure of feedback neural network is the same as single layer interconnection structure. And all these steps including data processing, data input and data output can be finished by every node of structure of feedback neural network. BP neural network and radial basis function neural network have been used widely in the field of feed-forward neural network. Hopfield neural network is the representative type among feedback neural network. For the great mass of feed-forward neural network has far superior ability for recognition and classification than feedback neural network, feed-forward neural network is applied to the study vehicle license plate identification technology of neural network based on genetic algorithm in this article.

Data signal flow of feed-forward neural network consists of function signal and error signal. Function signal, passed in from input terminal of the network, will be processed by neurons of hidden layer. And then it will be transferred to network output layer where it forms function signal propagating forward. Error signal will be generated when actual output is far away from expected output. Contrary to transfer direction of function signal, error signal will be transferred from network output layer to network hidden layer .At last, it will be transferred to network input layer where weight will be altered in order to make margin of error meet the origin requirements.

Feed-forward neural network consists of learning and recognition. Prepared information sample is to be transferred to feed-forward neural network step by step in the process of learning. There will be actual output data after being processed in network output layer and network hidden layer. Another information sample will be inputted if margin of error is in permeable limits after a comparison between the output data and expected data. If not, back propagation is needed and weight will be altered for the next learning 
step which is to be repeated as above. Information sample is to be transferred to feed-forward neural network in the process of recognition. Then there will be the result that is the unmistakable recognition with a high probability because waited recognition Information sample may be similar to what it have learned before and neural network is skilled in memory ability and associative capacity. Information sample can still be recognized correctly even if waited recognition Information sample is not similar to what it have learned before or there are noise and interference, because neural network is good at correction and fault tolerance. And whole characteristics of Information samples will be extracted by the network.

\section{Principle Introduction of Genetic Algorithm}

Genetic algorithm, first reported by Holland an American professor, is a kind of technique in search of optimizations by simulating the process of natural biological heredity and organic evolution. According to theory, genetic algorithm can seek out global optimal solution in the larger scale. New species can be generated because trans-mutated operation is applied to the algorithm. At present, BP neural network applied to recognition technology also has some weaknesses. For example, it is genetic algorithm based on the idea of gradient descent that tends to drop into the partial optimal solution. And convergence speed of the algorithm came a little slowly. Therefor, the research orientation with the combination of genetic algorithm and neural network has a profound significance, getting more and more attention.

During basic procedure of genetic algorithm, the first thing is to encode solution space of the problem in order to makes it become single individual. Then scale of initial population is to be designated before assigning fitness evaluation function. There will be output if single individuals meet the termination condition well. If not, a brand new single individual species will come into being, with the help of trans-mutated operation to these single individuals.

Next eminent single individuals will be copied from the first generation as the next generation. The Iterative work will be performed over and over again until global optimal solution meeting the demand appears.

Survival of the fittest is a natural feature of genetic algorithm which is a kind of global optimal searching algorithm based on Darwin's nature evolution theory and Mendel's genetics and mutation theory. It differentiates itself from traditional algorithm:

a.Problem parameters for coding are operands of genetic algorithm. Individual fitness serves as a criterion of judgment of genetic algorithm which can work out global optimal searching about high nonlinear problems.

b.Global optimal searching of genetic algorithm based on individual fitness is an oriented and simple algorithm rather than the exhaustion algorithm whose calculating quantity is excessive.

c.Its' good parallel features enable solutions collection to be their researching scope. Chances of dropping into the partial optimal solution will be greatly reduced because genetic algorithm avoids researching single individuals.

d.Global optimal searching is the greatest hallmark of genetic algorithm. With the help of trans-mutated operation, a brand new individual species will come into being.. By doing so, it can keep the diversity of population and enlarge the search scopes. As a result, it is beneficial to researching global optimal solution instead of partial optimal solution.

\section{Structure of Vehicle License Plate System}


Vehicle license plate recognition system designed in this article mainly contains three modules: license plate location, character segmentation and character recognition. Module of license plate location contains four aspects: image gray processing, image smoothing, image enhancement and license plate recognition. Module of character segmentation includes correcting position of license plate images, deleting border of license plate images and isolating character. At the same time, some efficient methods are provided for conglutination treatment. As for module of character recognition, it focuses on training and recognition based on neural network which is based on genetic algorithm.

\section{Principle of Neural Network Based on Genetic Algorithm Applied to Vehicle License Plate Recognition System}

There are many advantages of neural network, such as a high adaptability, good abilities of learning and the capacity for associational memory and tolerance of error etc. Feed-forward neural network based on $\mathrm{BP}$ algorithm has been wildly used when it comes to vehicle license plate recognition technique. But it's easy to drop into partial optimal searching so that global optimal solution is missing because BP algorithm is based on the idea of gradient descent. At the beginning, an initial value must be set according to empirical value .If the initial value isn't reasonable enough, convergence speed of the algorithm will slow down in return. Even worse, there is no convergence. Genetic algorithm, having the capacity for global optimal searching, improves the algorithmic efficiency by a long way and weakens the rigor of BP algorithm. Vehicle license plate system will acquire preferably effect due to the combination of the two algorithms.

Genetic algorithm consists of two processes: learning and recognition. The process of learning aims at researching of optimal network weight and threshold in order to keep error signal in the smallest scale. The process of learning will come to an end after gaining a global optimal solution.

Neural network based on genetic algorithm regards genetic algorithm as an algorithm for learning of process. Firstly, weight and threshold will be coded in hidden layer of neural network in order to turn it into chromosomes in the genetic network space. And then chromosomes will be optimized using trans-mutated operation of neural network trans-mutated operation. Evolutionary process will come to an end when minimum error signal value between actual output weight and threshold of neural network and expected output one is seeked out.

\section{Conclusions}

There are various vehicle license plate recognition systems. System based on BP algorithm has been wildly used but it is based on the idea of gradient descent that tends to drop into the partial optimal solution. In order to solve the problem, genetic algorithm and neural network are combined together in this article. The research on the vehicle license plate recognition of computer system to which both global optimal researching of genetic algorithm and implementing advantages of neural network are applied presents satisfying results.

\section{References:}

[1] Y.R.Liu, Z.H.Zhang, L.S.Liu. A Study And Emulation Based on BP Neural Network Algorithm PID Controller[J](In Chinese). Electronic Design Engineering, 2012(12):140-142

[2] F.Q.Guo. Analog Fault Diagnosis Based on Wavelet Neural Network and Phase Difference [J] (In Chinese).Modern electronic technique, 2012(13):183-186. 
[3] R.J.Wu. Research of Neural Network Applied to Safety Assessment of Computer Network.[J]. (In Chinese) Computer Emulation, 2011(11):126-129. 